\title{
The amphibian microbiome exhibits poor resilience following pathogen-induced disturbance
}

\author{
Andrea J. Jani $\mathbb{D}^{1,2} \cdot$ Jessie Bushell ${ }^{3} \cdot$ Cédric G. Arisdakessian $\mathbb{1}^{4} \cdot$ Mahdi Belcaid $^{4} \cdot$ Daniel M. Boiano ${ }^{5}$. \\ Cathy Brown ${ }^{6} \cdot$ Roland A. Knapp $\mathbb{D}^{7}$
}

Received: 18 August 2020 / Revised: 24 November 2020 / Accepted: 7 December 2020 / Published online: 9 February 2021

(c) The Author(s) 2021. This article is published with open access

\begin{abstract}
Infectious pathogens can disrupt the microbiome in addition to directly affecting the host. Impacts of disease may be dependent on the ability of the microbiome to recover from such disturbance, yet remarkably little is known about microbiome recovery after disease, particularly in nonhuman animals. We assessed the resilience of the amphibian skin microbial community after disturbance by the pathogen, Batrachochytrium dendrobatidis (Bd). Skin microbial communities of laboratory-reared mountain yellow-legged frogs were tracked through three experimental phases: prior to Bd infection, after Bd infection (disturbance), and after clearing Bd infection (recovery period). Bd infection disturbed microbiome composition and altered the relative abundances of several dominant bacterial taxa. After Bd infection, frogs were treated with an antifungal drug that cleared Bd infection, but this did not lead to recovery of microbiome composition (measured as Unifrac distance) or relative abundances of dominant bacterial groups. These results indicate that Bd infection can lead to an alternate stable state in the microbiome of sensitive amphibians, or that microbiome recovery is extremely slow-in either case resilience is low. Furthermore, antifungal treatment and clearance of Bd infection had the additional effect of reducing microbial community variability, which we hypothesize results from similarity across frogs in the taxa that colonize community vacancies resulting from the removal of Bd. Our results indicate that the skin microbiota of mountain yellowlegged frogs has low resilience following Bd-induced disturbance and is further altered by the process of clearing $\mathrm{Bd}$ infection, which may have implications for the conservation of this endangered amphibian.
\end{abstract}

Supplementary information The online version of this article (https:// doi.org/10.1038/s41396-020-00875-w) contains supplementary material, which is available to authorized users.

Andrea J. Jani

jania@hawaii.edu

1 Department of Oceanography, Center for Microbial Oceanography: Research and Education (CMORE), University of Hawai'i at Mànoa, Honolulu, HI, USA

2 Pacific Biosciences Research Center, University of Hawai'i at Mànoa, Honolulu, HI, USA

3 San Francisco Zoological Society, San Francisco, CA, USA

4 Information and Computer Sciences, University of Hawai'i at Mànoa, Honolulu, HI, USA

5 Sequoia and Kings Canyon National Parks, National Park Service, Three Rivers, CA, USA

6 USDA Forest Service, Stanislaus National Forest, Sonora, CA, USA

7 Sierra Nevada Aquatic Research Laboratory, University of California, Mammoth Lakes, CA, USA

\section{Introduction}

Ecological communities exist in dynamic environmental landscapes, and experience changes ranging from regular incremental shifts such as light and nutrient gradients to large scale disturbances such as disease outbreaks or storm events. Disturbance can alter the structure of ecological communities: it can maintain long term community diversity [1], but can also degrade community or ecosystem function [2-4]. Understanding what enables a community to maintain stable structure or function in the face of disturbances is a fundamental aim of ecology, and has increasing practical importance as human activities alter the frequency and intensity of disturbances such as fire, storms, drought, and disease outbreaks [5].

Community stability comprises resistance and resilience. Resistance is the capacity for a community to resist change due to disturbance. In contrast, resilience is the rate at which a community recovers to its initial state after a disturbance has occurred, and is measured as either the degree of 
recovery at a given time point or the time required for complete recovery [6]. A growing number of studies has examined resistance (or its converse, sensitivity) to disturbance, but far fewer studies have explicitly tested resilience [6]. Studies so far have found that resilience often varies among study systems. For example, soil communities often fail to return to baseline structure [7], while lake microbial communities showed complete recovery $[8,9]$. Among host-associated microbial communities, resilience has arguably been most studied in humans, particularly the human gut. The human gut microbiome showed partial recovery following a variety of perturbations, including antibiotics [10, 11], severe diarrhea [12], and diet interventions [13]. Studies of microbiome resilience in nonhuman animals are rare [6], but see $[14,15]$.

Exposure of a host to an infectious pathogen can represent a disturbance to the microbiome [16-18]. However, little is known about the resilience (recovery) of nonhuman animal microbiomes following disturbance by infectious disease. Batrachochytrium dendrobatidis (Bd) is a chytrid fungus that infects the skin of amphibians and causes the potentially lethal disease chytridiomycosis [19]. Since its discovery just over two decades ago, Bd has emerged as a global threat to amphibians, affecting hundreds of amphibian species and driving massive population declines [20-23]. The need to control the disease has spurred research into possible treatments, including vaccination, control of reservoir hosts, and probiotics [24-26]. Research on probiotic efficacy has yielded variable results: bacteria isolated from amphibian skin inhibited Bd growth or infection in some cases (e.g., [27, 28], but not others [29, 30]). Understanding the stability of the amphibian skin microbiome is relevant to probiotic success or failure for two reasons. First, $\mathrm{Bd}$ infection disturbs the microbiome, indicating that disease mitigation strategies may benefit from the ability to improve microbiome stability [17]. Second, and somewhat paradoxically, microbiome stability may hinder establishment of potentially beneficial probiotics, a phenomenon sometimes referred to as colonization resistance. Understanding of the factors that shape and alter the amphibian microbiome has grown considerably in recent years. Several studies have examined the factors associated with amphibian microbiome composition and diversity (e.g., [31-39]). In addition, studies have experimentally measured effects of disturbances, including $\mathrm{Bd}$ or viral infection [17, 40-42] and antibiotic exposure [43], on the microbiome. From these studies, it is clear that Bd infection can disturb the microbiome. However, data on the capacity for the microbiome to recover from such disturbances are extremely rare.

In this study, we assess the recovery, after disturbance by $\mathrm{Bd}$, of amphibian-associated microbial communities (also referred to as microbiota). Our focal host species is the mountain yellow-legged frog (Rana muscosa). Rana muscosa forms a species complex with Rana sierrae, and both are endemic to the Sierra Nevada and severely threatened by Bd [23, 44]. Bd infection disturbs the microbiome of these frogs [17], and as such understanding microbiome resilience in this species is particularly important. In this study, we exposed mountain yellow-legged frogs to $\mathrm{Bd}$ (or sham inoculum) and later cleared frogs of infection using an antifungal drug. We present data on Bd infection and microbiome composition and diversity before infection, during the infected stage, and after clearance of the pathogen to test if clearing frogs of Bd leads to recovery of the original microbiome structure.

\section{Materials and methods}

\section{Ethics statement}

Collection and handling of $R$. sierrae and $R$. muscosa was planned so as to minimize impacts to individuals or populations. Captures were part of a conservation effort and not conducted solely for research purposes. Work was conducted under permits from the US Fish and Wildlife Service, National Park Service, and CA Department of Fish and Wildlife, with approval from the UCSB and UC Davis IACUC and the San Francisco Zoo Research Review Committee. See Supporting Materials for permit numbers.

\section{Study design}

This study was part of an effort to restore $R$. muscosa and $R$. sierrae populations through "head-starting" (captive rearing followed by release to the wild). Eighty-seven out of 125 frogs were exposed to Bd in an attempt to immunize against future infection [26], and then cleared of $\mathrm{Bd}$ using an antifungal drug, prior to release. A random subset of 38 frogs served as controls and were not exposed to Bd. Skin microbiome swabs were collected from a subset of frogs at three time points: pre-infection (at the start of the experiment, before Bd exposure), post-infection (1 week after Bdexposure), and recovery period (3 weeks after treating frogs with an antifungal drug to clear Bd infections). Swabs to quantify Bd infection were collected approximately weekly. The study ended 48 days after $\mathrm{Bd}$ exposure. The entire study was conducted in the laboratory, before frogs were rereleased to the wild.

\section{Frog populations and handling}

Frogs in this study came from the Sierra Nevada mountains, California, USA (Table S1A). Four source populations of $R$. muscosa are located in the southern Sierra (Sequoia and Kings Canyon National Parks), and recently suffered Bdinduced declines. A fifth population ( $R$. sierrae, from 
Plumas National Forest in the northern Sierra) was included in the conservation (head-starting) effort but is excluded from this study because all Plumas frogs were exposed to $\mathrm{Bd}$ (there were no control frogs from Plumas) due to conservation priorities. However, for completeness an analysis comparing microbial communities between Plumas and the four $R$. muscosa populations is provided in the supporting Materials.

Frogs were collected as tadpoles or metamorphs using hand nets and transported from field sites to vehicles in 41 plastic jugs filled with lake water and equipped with aerators (tadpoles) or in individual $60 \mathrm{ml}$ plastic containers containing $\sim 10 \mathrm{ml}$ of lake water (metamorphs). Animals were transported to the San Francisco Zoo, where they were housed in tanks with tap water purified by reverse osmosis and supplemented with Kent Marine R/O Right. Collections took place in the summers of 2015 and 2016. Animals were reared in captivity for 1-2 years to ensure acclimation to the lab and allow all animals to complete metamorphosis prior to beginning the study. (Rana muscosa is a long-lived species: in the wild, development from egg through metamorphosis generally takes 2-3 summers (about 1-2 years), and total life spans of 9-12 years are common (Knapp, unpublished). All animals in this study were adults or latestage sub-adults and were 2-3 years old. Previous research with both Rana sierrae and a close relative (Rana cascadae) found no difference between the microbiomes of adults and subadults [17, 34]. For this study, frogs were housed in groups of 3-6 individuals per tank, with Bd-infected and uninfected frogs housed in separate rooms as a precaution against cross-contamination. Individual frogs were identifiable by unique passive integrated transponder (PIT) tags. Frogs were fitted with PIT tags either 1 year or 5-6 days prior to the start of the experiment. PIT tag date did not differ between experimental treatment groups (Chi Square $P=0.311)$. Tank water was changed daily.

\section{Bd exposures (immunizations)}

Sierra Nevada Bd isolates were cultured on tryptone agar and $\mathrm{Bd}$ inocula and sham inocula were prepared as described in [33], using the same Bd strains. On each of 3 consecutive days, $10^{6}$ live zoospores $(250,000$ of each of the four strains), or a sham inoculum (prepared from agar media plates without Bd), was added to each frog tank. Tank water was not changed during the 3-day inoculation period.

\section{Itraconazole treatment}

Three weeks after $\mathrm{Bd}$ infection, frogs were treated with itraconazole for 6 days to clear Bd infection [45]. Itraconazole treatment is a common method for clearing amphibians of Bd infection [46], and has been used by our group to treat $\mathrm{Bd}$ infection in $R$. muscosa. All frogs (infected and uninfected) were treated with itraconazole to control for any side effects of the drug.

\section{Swab collection and processing}

Skin-associated microbes were collected using sterile synthetic swabs as described previously [33]. Frogs were rinsed with sterile water prior to swabbing. Bd swabs were collected 1 day prior to $\mathrm{Bd}$ exposure and approximately weekly for the duration of the experiment (48 days). Bd swabs were collected from all frogs $(N=125)$. Microbiome swabs were collected from a subset of 59 frogs (41 Bd-exposed, 18 unexposed) on three dates: immediately before $\mathrm{Bd}$ exposure, 1 week after Bd exposure, and 3 weeks after antifungal treatment ( 7 weeks after Bd exposure). When swabbing any given frog, we always collected the microbiome swab first and the $\mathrm{Bd}$ swab second because pilot data showed no significant difference in $\mathrm{Bd}$ loads obtained from first or second swabs (Jani, unpublished). DNA was extracted from swabs, including negative controls, using the Prepman Ultra reagent (Applied Biosystems) as described previously [47].

\section{Quantification of Bd infection}

Bd infection was quantified from swab DNA by quantitative PCR (qPCR) using a probe-based (Taq-Man, Applied Biosystems) assay that targets the internally transcribed spacer (ITS) region, with conditions, primers, and probe sequences following [48]. A 5-point standard curve was included on each plate (range of $10^{2}-10^{6}$ ITS copies). Bd load data are in units of ITS gene copies per swab, and are log10-transformed.

\section{Sequencing library preparation}

We prepared a multiplexed library for Illumina sequencing following Kozich [49]. Briefly, indexed oligonucleotides 341F (CCTACGGGNGGCWGCAG) and 785R (GACTAC HVGGGTATCTAATCC) were used to amplify the V3-4 region of the 16S rRNA gene [50]. PCR products were purified using a PCR Purification and Normalization kit (Charm Biotech), then pooled in equimolar quantities. The library was sequenced on one lane of an Illumina MiSeq with v3 chemistry and 300 paired end cycles.

\section{Bioinformatic processing of Illumina sequence reads}

We pre-processed raw sequence data into exact (amplicon) sequence variants (ASVs) at the $100 \%$ nucleotide identity using the dada $2 \mathrm{R}$ package [51]. Reads were truncated at position 260/190 (forward/reverse read). Reads were discarded using the filterAndTrim() function if they contained 
one or more bases with quality scores $<2$, or more than 3 expected errors. Denoising was performed with the learnError() and dada() functions with default parameters. Reads were merged using the mergePairs() function, and any pairs with an overlap of fewer than 20 bases, or with more than one mismatch, were discarded. We used mothur v1.42.3 [52] and the Silva (release 132) database [53] to align and annotate sequences. Sequences with a start or stop position outside the 5-95th percentile range (over all sequences) were discarded. We removed potential chimeras with chimera.vsearch(). Taxonomies were assigned using classify.seqs() and classify.otus(). We removed all mitochondrial or chloroplast ASVs, as well as sequences with no annotations at the domain level. We standardized the number of sequences across all samples by subsampling 5000 reads per sample using sub.sample(). Samples fewer than 5000 reads were discarded. ASVs were defined as unique "amplicon sequence variants" by dada2; we used the lulu R package [54] to refine ASVs as follows: we merged ASVs if all of the three following conditions were satisfied: (1) They co-occur in every sample, (2) One of the two ASVs has a lower abundance than the other in every sample and (3) they share a sequence similarity of at least $97 \%$. Finally, we discarded ASVs with a total abundance of two or fewer reads. Pipeline code is available from [55]. Relative abundance data were arc-sine(square root) transformed. Table S1B shows the final number of samples in each treatment and time point after bioinformatic quality control.

\section{Microbiota analysis: overview}

For clarity, we refer to the treatment groups that were exposed or not exposed to $\mathrm{Bd}$ as "Bd+" and "Bd-", respectively. Note that the $\mathrm{Bd}+$ treatment is referred to as $\mathrm{Bd}+$ even before frogs were exposed to $\mathrm{Bd}$. We use the terms pre-infection, post-infection, and recovery period to indicate the time periods (phases of the experiment) before frogs were exposed to $\mathrm{Bd}$ (or to sham inoculum), after frogs were exposed to $\mathrm{Bd}$, and after itraconazole treatment, respectively.

\section{Measuring microbial community disturbance}

Community change in response to disturbance can be measured in terms of change from an initial baseline. In this study we observed large changes in the microbiota over time, even in control frogs. To tease apart change due to $\mathrm{Bd}$ infection from temporal variation due to unknown causes, we measured Bd-induced microbial community shift as the difference between treatment and control frogs at a given time point. We determined the effect of $\mathrm{Bd}$ infection and Bd-clearance on microbiome composition, multivariate dispersion, and diversity as follows:

\section{Composition}

We measured turnover in microbial community composition (beta diversity) using weighted Unifrac distances [56]. We used NMDS ordination to display whole-community similarity among samples. To test if community composition differed among groups, we ran permutational multivariate analysis of variance (PERMANOVA, [57, 58]) using the adonis function (Vegan package) in R, with 999 permutations. We first ran PERMANOVA on all data together, with $\mathrm{Bd}$ treatment $(\mathrm{Bd}+$ or $\mathrm{Bd}-$ ), frog source population, frog tank, time period, PIT tag group, and the treatment*time and population*time interactions as predictors. We then ran the PERMANOVA model (without time as a predictor) separately for each time phase of the experiment because we are primarily interested in differences between treatments within each time point.

We used LEfSe [59] to identify bacterial taxa that respond to $\mathrm{Bd}$ infection or Bd clearance. LEfSe analyses were done separately for each time point, with Bd treatment as class, source population as subclass, standardized abundances, restriction of pairwise comparisons to within subclass, and defaults for all other settings. We also explored whether taxa affected (or unaffected) by Bd tend to be numerically dominant by identifying ASVs that were both widespread (present in both treatments, at all time points) and abundant (cumulatively accounting for over $90 \%$ of all sequence reads). We refer to this set of ASVs as "core" taxa for brevity, noting that the thresholds are arbitrary and not intended to suggest a functional core.

\section{Dispersion}

To test for differences in multivariate dispersion (i.e., amongfrog variability) of community composition, we used PermDISP (betadisper function in the Vegan R package), followed by the permutest function to determine statistical significance. PermDISP was performed separately for each time point (comparing $\mathrm{Bd}+$ to $\mathrm{Bd}-$ ) as well as for all time points combined. Frog population was not included in dispersion analysis because the test does not accommodate multiple predictor variables.

\section{Alpha diversity}

We tested for treatment effects on alpha diversity using linear mixed effects models with $\mathrm{Bd}$ treatment, frog population, time point, PIT tag group, treatment*time and population*time as predictors, tank as a random effect, and diversity metrics (observed richness, Chao's richness, Shannon diversity, and Shannon evenness) as response variables. As for community composition analyses, we first ran the model on all data together, and followed with a separate analysis for each time point of the experiment. 


\section{Visualizing disturbance}

Resistance and resilience can be visually represented by plotting the magnitude of disturbance through time (e.g., Shade et al. 2012, [6]). Following this framework, we plotted the distance of the $\mathrm{Bd}+$ frog microbiomes relative to Bd- controls at each time point. For visualization, the difference between the microbial communities of $\mathrm{Bd}+$ and $\mathrm{Bd}-$ frogs was calculated as the mean Unifrac distance between $\mathrm{Bd}+$ and $\mathrm{Bd}-$ frogs at each time point. (Details in Supporting Materials.)

\section{Predictors of stability}

We explored potential drivers of microbial community stability by testing if factors that vary at the start of the experiment affect stability later in the experiment. Specifically, we used linear regressions to test if the magnitude of community change through time (measured as Unifrac distance) for each frog is affected by initial alpha diversity or PIT tag group. We also asked if individual frogs have inherent differences in microbial community stability by using linear regression to test if stability (magnitude of microbiome shift) during the infection phase predicted stability through the recovery phase. (Details in Supporting Materials.)

\section{Effects on host physiology}

To test if Bd-induced disturbance of the microbiota was linked to changes in frog physiological condition, we measured changes in frog body mass over the course of the study, using two metrics: proportional mass change ([mass final $\left.-\mathrm{mass}_{\text {initial }}\right] /$ mass $\left._{\text {initial }}\right)$ and change in mass adjusted for body length ([mass final mass $\left._{\text {initial }}\right] /$ snout-vent length). We used linear regression to test if mass change was predicted by the magnitude of microbial community shifts (measured as Unifrac distance between two time points for each frog).

\section{Results}

\section{Microbiome diversity and composition}

PERMANOVA of all time points together revealed effects of Bd treatment, population, time period, population*timeperiod interaction and $\mathrm{Bd} *$ time-period interaction (PERMANOVA, $P<0.05$, Table S2). Tank and PIT tag group had no significant effects. The $\mathrm{Bd} *$ time interaction suggests an effect of Bd treatment in some time points but not others, as one might expect given that the "Bd+" group was not yet infected in the pre-infection period. Alpha diversity also varied by time period $(P<0.05$, Table S3) but not $\mathrm{Bd}$ treatment, population, or PIT tag group.
We then analyzed each time point separately to focus on between treatment differences within each time point. At the start of the experiment (pre-infection), there were no differences between treatment groups in any metrics of microbiome structure $(P>0.05$ for tests on alpha diversity, community composition, and multivariate dispersion; Fig. 1A, Tables S4 through S7). Raw relative abundances of common bacterial taxa, for every frog at every time point as well as averaged by time point and treatment, are shown in Figs. S1 and S2.

$\mathrm{Bd}$ infection altered microbiome composition on frogs: post-infection, the microbiota of $\mathrm{Bd}+$ and $\mathrm{Bd}-$ frogs differed in composition (PERMANOVA, $P<0.001$, Fig. 1B, Table S6) but not alpha diversity $(P>0.05$ for all metrics, Table S7) or dispersion (BETADISPER $P=0.880$, Fig. 1B, Table S4).

Itraconzole treatment cleared frogs of Bd infection (Fig. 2, Fig. S3) but did not reverse effects of $\mathrm{Bd}$ on microbiome composition: microbiota of $\mathrm{Bd}+$ and $\mathrm{Bd}-$ treatment groups still differed during the recovery period (PERMANOVA, $P<$ 0.001, Fig. 1C, Table S6). In addition, clearing frogs of $\mathrm{Bd}$ reduced multivariate dispersion of community composition for $\mathrm{Bd}+$, but not $\mathrm{Bd}-$, frogs. This is demonstrated by a significant effect of $\mathrm{Bd}$ treatment on dispersion after $\mathrm{Bd}$ clearance (during the recovery period), and not before $\mathrm{Bd}$ clearance (Fig. 1C, D. betadisper, $P=0.013$ for recovery phase, $P=0.880$ for post-infection). Further analysis of dispersion among all pairwise time*treatment comparisons (pairwise betadisper, all time points included) further showed that the $\mathrm{Bd}+$ /recovery phase group had lower dispersion than any other group. Clearing Bd had no effect on alpha diversity ( $P>0.05$ for all metrics, Table S7).

Frog source population had a significant effect on microbiome composition at the post-infection and recovery time points (PERMANOVA, $P<0.001, P=0.005$, Table S6), but not prior to $\mathrm{Bd}$ infection $(P=0.145)$.

For completeness, we reran the above PERMANOVA models with unweighted Unifrac and Bray-Curtis metrics. Results were qualitatively the same as for weighted Unifrac: Bd treatment was not significant pre-infection (unweighted Unifrac $P=0.170$, Bray-Curtis $P=0.411$ ), and was significant post-infection $(P<0.001, P<0.001)$ and during the recovery period $(P<0.001, P=0.002)$.

\section{Bd infections}

Figure 2 shows $\mathrm{Bd}$ infection loads over the course of the experiment. All $\mathrm{Bd}+$ frogs became infected by 1 week after being exposed to $\mathrm{Bd}$, with mean Bd load of 67,546 ITS copies per frog). $\mathrm{Bd}+$ frogs remained infected throughout the post-infection period ( 3 weeks). Of the 275 swabs collected from $\mathrm{Bd}-$ frogs (prior to infection or from the $\mathrm{Bd}-$ treatment group), six returned positive qPCR results. These were deemed to be false positives because all swabs 
A

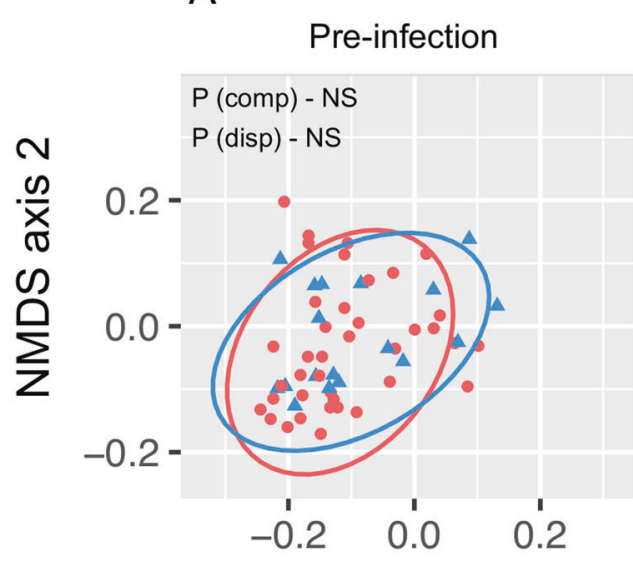

B

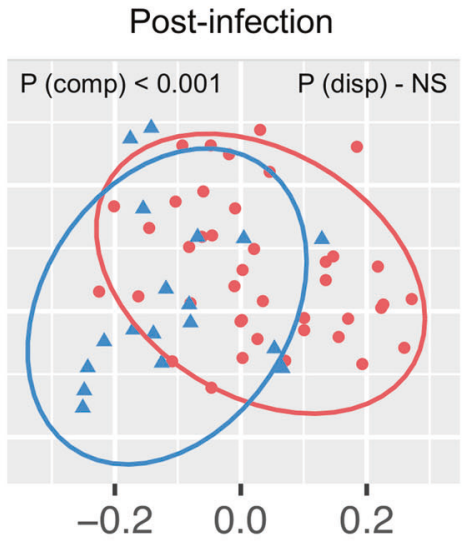

NMDS axis 1
C

\section{Recovery period}

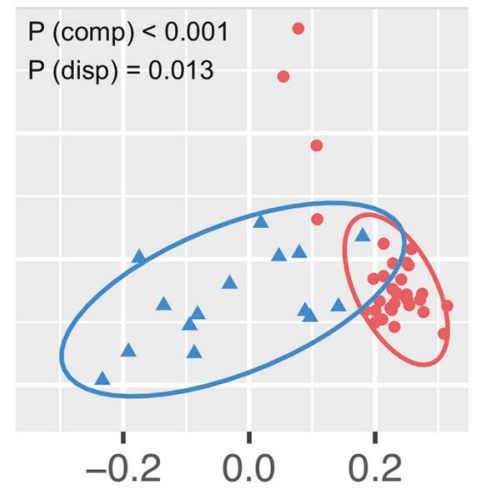

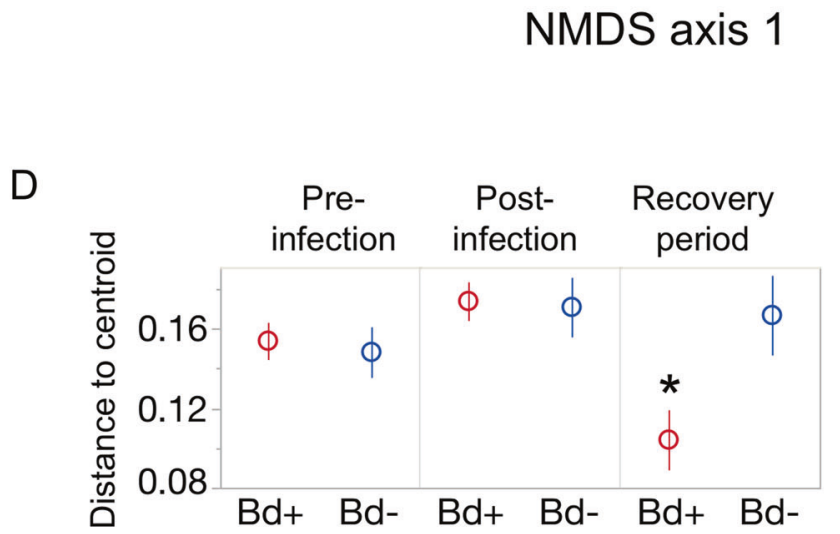

Fig. 1 Changes in community composition and dispersion through time. Top panel shows NMDS ordination of microbial communities: A Pre-infection, no difference between treatments. B Post-infection: composition (position of ellipses) but not dispersion (sizes of ellipses) differed between treatments. C Recovery period: Clearing Bd infection did not lead to microbial community recovery. Bd-clearance homogenized the microbiota of Bd-infected (but not uninfected) frogs. Ellipses are $95 \%$ normal data ellipses. D Microbial community

collected at time points before or after the equivocal swabs from Bd- frogs were PCR-negative, and the Bd loads were very low-below 5 gene copies per qPCR reaction. For comparison, a single Bd cell from Sierra Nevada strains is estimated to have $\sim 60$ ITS gene copies [60]. Bd load was not affected by PIT tag group or population (Repeated Measures ANOVA, PIT $*$ time interaction: $P=0.901$, population*time interaction: $P=0.056$ ).

\section{Microbial community displacement through time}

Clearing frogs of $\mathrm{Bd}$ infection did not lead to microbial community recovery: the difference between $\mathrm{Bd}+$ and $\mathrm{Bd}-$ frogs was not reduced (Figs. 2, 3). The proportion of microbial community variation explained by $\mathrm{Bd}$ treatment was greater after $\mathrm{Bd}$ clearance than it had been during infection (PERMANOVA, $R^{2}$ of Bd effect: $0.12,0.37$ for post-infection and recovery period, respectively). dispersion: mean Unifrac distance (within each treatment-by-time group) of individual microbial communities to the group centroid. Asterisk indicates a group that differs significantly from all other groups ( $P$ values: Table 5). Error bars are standard error. NMDS was performed on all data together, then separated by experiment phase $(\mathbf{A}, \mathbf{B}, \mathbf{C})$ for clarity. $P$ values correspond to PERMANOVA (comp) and PERMDISP (disp) tests. Ordination stress: 0.06.

\section{Bacterial taxa affected}

Bd infection altered the relative abundance of several bacterial taxa (Figs. 4, 5, and S4; Table S8). Figure 4 shows raw relative abundances, but note that LEfSe employs nonparametric rank-based (Kruskal-Wallis) tests, which are less sensitive to the magnitude of differences. LEfSe maintains low false positive rates with the requirement that any difference be consistently detected in all sub-classes (populations) of the data. Bd infection caused the "genus" Undibacterium and an ASV classified to Weeksellaceae as well as the entire family (phylotype) Weeksellaceae to increase relative to controls (Fig. 4). ASVs classified as Rubritaleaceae, Stenotrophomonas, and Verrucomicrobiales declined (Fig. 4). For Stenotrophomonas, the difference between treatment and control was statistically significant but very small. One ASV (ASV421, classified as to Burkholderiaceae) appeared to increase in control $(\mathrm{Bd}-)$ frogs, 


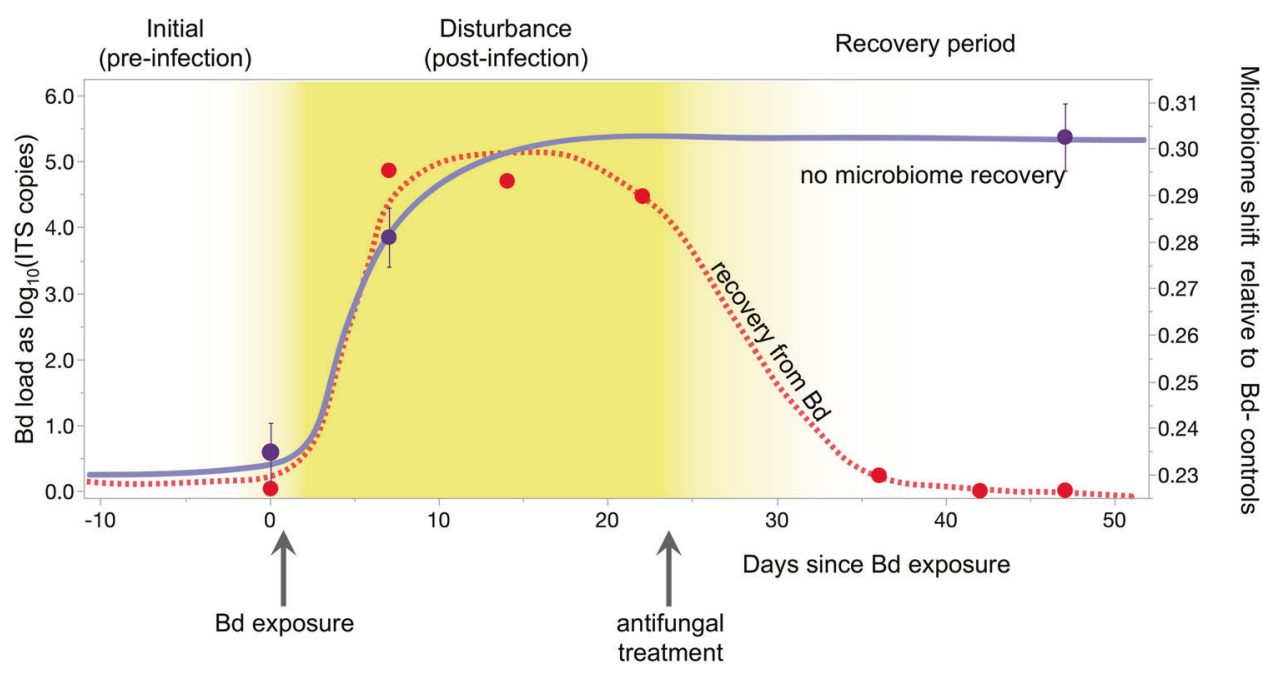

Fig. 2 Shifts in Bd infection intensity and microbial community composition through time. Left axis (red symbols): Mean Bd loads through time for $\mathrm{Bd}+$ frogs, showing clearance of $\mathrm{Bd}$ after itraconazole treatment. Bd loads are expressed as the number of Bd ITS copies detected per frog, on a $\log _{10}$ scale. Right Axis (purple symbols): Shift in microbial communities of $\mathrm{Bd}+$ frogs relative to $\mathrm{Bd}-$ controls, measured as mean Unifrac distance between treatment and control. Curved lines are a conceptual diagram following [6], superimposed to visually connect time points for $\mathrm{Bd}$ load (red) and microbiome (purple). Error bars indicate standard error. Error bars for Bd load are not visible because they are smaller than size of the circle symbols; See Fig. S3 for box plots of Bd infection loads.

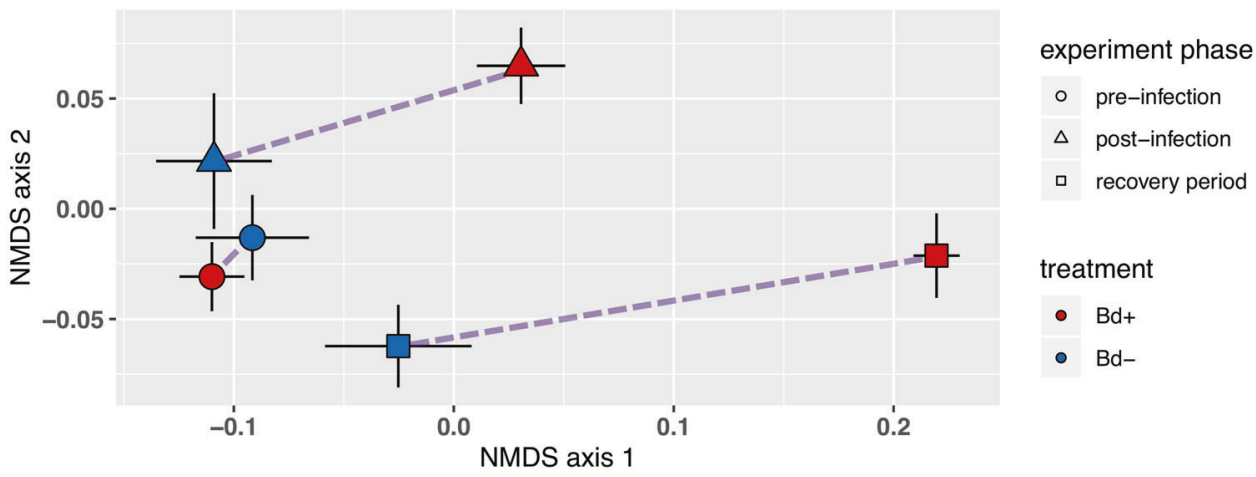

Fig. 3 NMDS ordination illustrating microbial community displacement of the $\mathrm{Bd}+$ group relative to the $\mathrm{Bd}-$ group in each phase of the experiment. Symbols indicate group mean and standard

but this may be a spurious finding because ASV421 was very rare (median relative abundance 0 in exposed and 0.0005 in $\mathrm{Bd}-$ frogs), which may make the analysis of this ASV sensitive to the limit of detection.

Clearing Bd with itraconazole did not reverse the effects of $\mathrm{Bd}$ on the most dominant taxa within the time frame of the experiment, although some less abundant taxa did recover. Weeksellaceae and Rubritaleaceae (both dominant members of the microbiota), and ASVs classified to Verrucomicrobiales did not recover (Fig. 4). Furthermore, Weeksellaceae and Rubritaleaceae continued to increase or decrease, respectively, in $\mathrm{Bd}+$ frogs even after $\mathrm{Bd}$ clearance. In contrast, the Undibacterium and Stenotrophomonas appeared to recover after $\mathrm{Bd}$ clearance: they no longer error of NMDS coordinates from Fig. 1, shown here with all three time points in one plot. Purple dashed lines indicate displacement between treatment and control groups within each time period.

differed between treatment groups (Fig. 4). Xanthomonadaceae increased slightly in $\mathrm{Bd}+$ frogs after clearance of $\mathrm{Bd}$ infection (Fig. S4). None of the taxa affected by Bd differed prior to $\mathrm{Bd}$ infection.

\section{Core bacterial taxa}

Eleven ASVs comprised the "core" microbiome. These included taxa affected by Bd, (Rubritaleaceae, Weeksellaceae, Stenotrophomonas, and an ASV classified as Undibacterium). The remaining core ASVs appeared stable (not significantly affected by Bd infection), and were classified to Pseudomonas (3 ASVs), Gracilibacteria (2), Enterobacteriaceae (1), and Burkholderiaceae (1). 
Bacteria that did not recover:

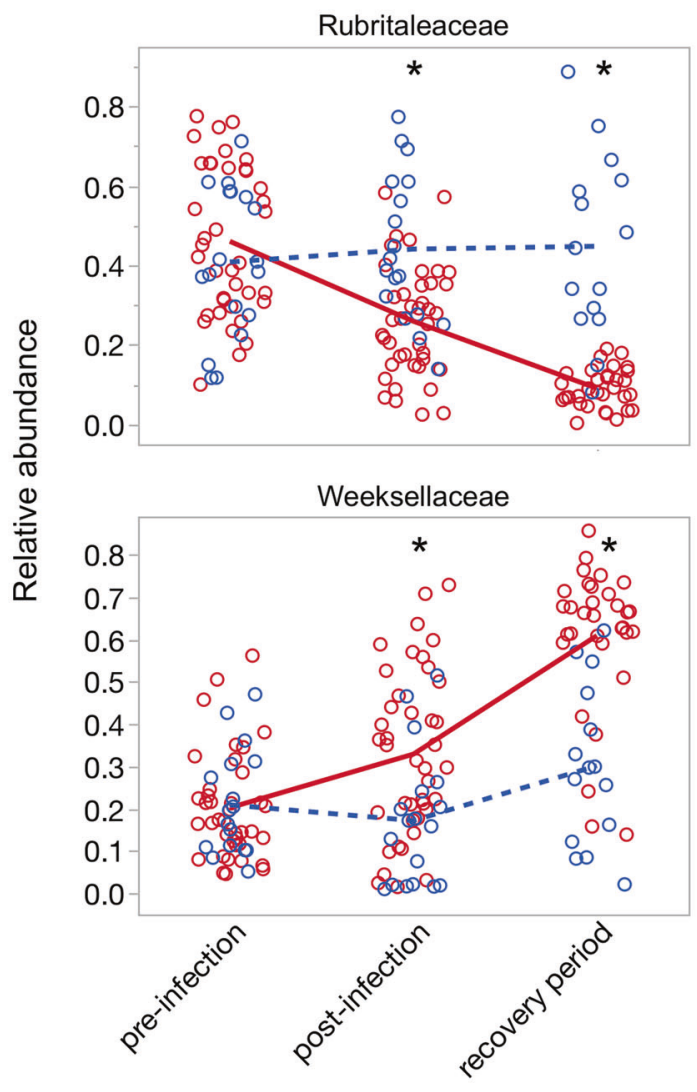

Bacteria that recovered:
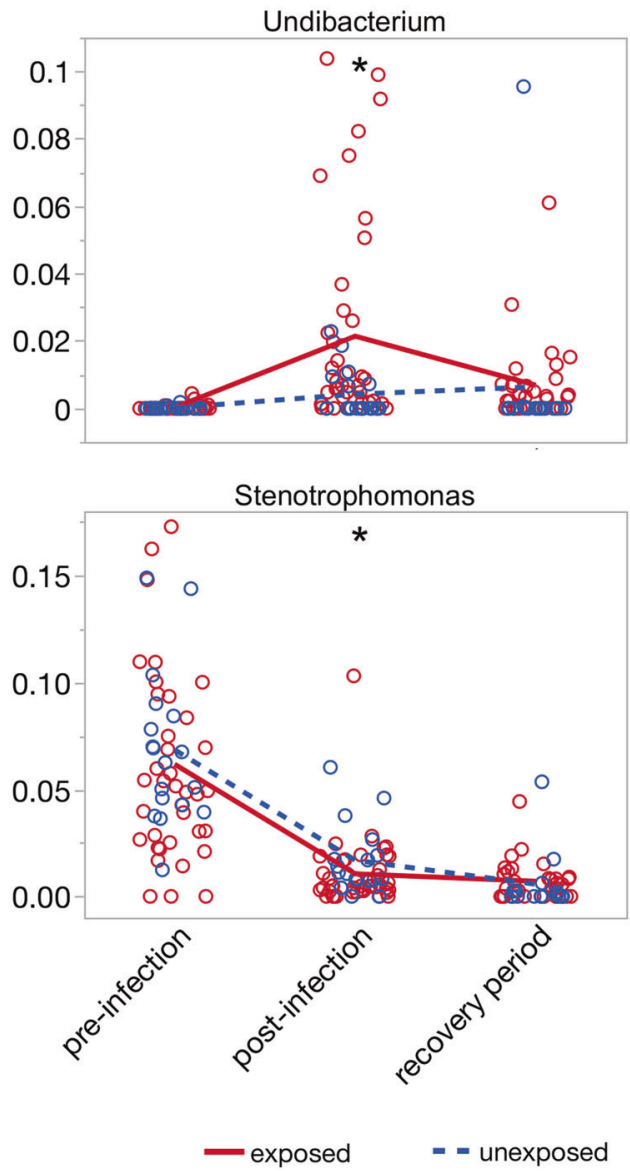

Fig. 4 Change in abundance through time for bacterial taxa affected by Bd. Bacterial taxa shown in left panels recovered after Bd clearance, while taxa shown in right panels did not. Plots show raw relative abundance data while statistics are rank-based (LEfSe).

\section{Predictors of microbial community stability}

Microbial community stability, (the magnitude of temporal change, measured as Unifrac distance,) was not predicted by alpha diversity or PIT tag group (linear regression, $P>0.05$ for all four metrics of alpha diversity; PIT tag group $P=$ 0.168). Individual frogs did not appear to have inherent stability levels: stability during the first phase of the experiment (shift from pre- to post-infection) did not predict stability during the second phase (shift from post-infection to recovery period, linear regression, $P=0.927$ ).

\section{Frog physiology}

Body mass change was negatively correlated with the shift in the microbiota (Unifrac distance) from pre-infection to post-infection $(P=0.010, P=0.023$ for proportional mass change and length-adjusted mass change, respectively). When analyzing the $\mathrm{Bd}+$ and $\mathrm{Bd}-$ treatments separately, microbiota shift predicted weight loss in the $\mathrm{Bd}+$ group
$(P=0.005, P=0.016)$, but not the $\mathrm{Bd}-$ group $(P=0.474$, $P=0.097)$, consistent with the expectation that microbial changes in the $\mathrm{Bd}-$ group are due to temporal drift, unassociated with directional changes in host condition.

\section{Discussion}

The maintenance of ecological community function through time depends on community resistance and resilience in the face of disturbances. Although Bd infection has been shown to disturb the microbiome, very little is known about resilience following such disturbance. We found that $\mathrm{Bd}$ infection altered the microbiota as expected, but clearing infection did not lead to recovery of the original bacterial community structure. Microbial communities did not regain their original composition, nor did Bd clearance reduce the magnitude of displacement between the microbial communities of Bd-exposed and control frogs. Examining changes in relative abundances of individual bacterial ASVs, we 
Fig. 5 Cladogram of bacterial taxa that differed between Bdinfected and uninfected frogs. Blue stars in legend indicate taxa that remained different after clearance of Bd infections. Cladogram constructed in the program LEfSe.

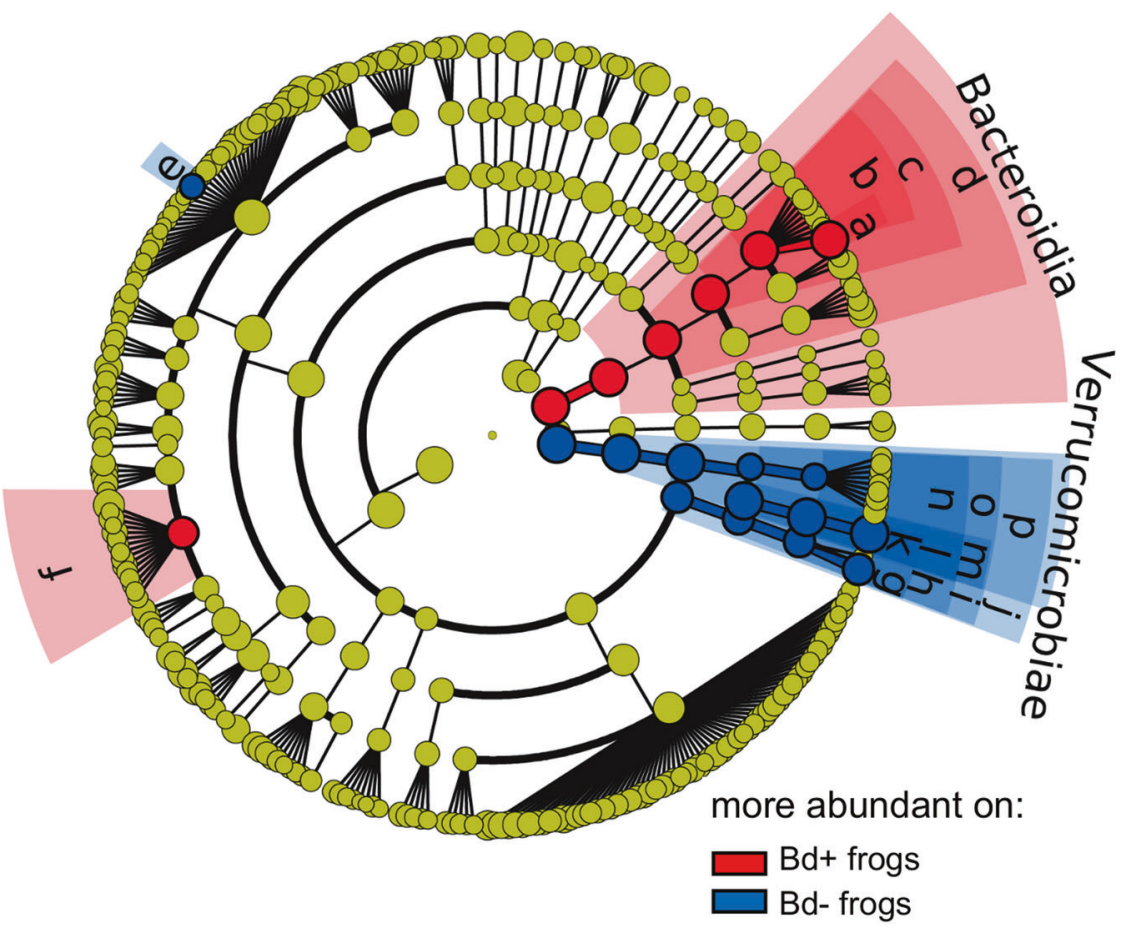

LEGEND: Taxa affected by Bd infection

(d) Flavobacteriales - (c) Weeksellaceae - (b) unclassified - (a) ASV0002 לे

(e) ASV0421 (Burkholderiaceae - Acidovorax)

(f) Undibacterium (phylotype)

(j) Xanthomonadales- (i) Xanthomonaaceae - (h) Stenotrophomonas - (g) ASV0004

(p) Verrucomicrobiales - (o) unclassified - (n) unclassified

(p) Verrucomicrobiales - (m) Rubritaleaceae - (l) unclassified - (k) ASV0001 하 found that the most abundant bacterial groups did not recover (Weeksellaceae and Rubritaleaceae), although some less abundant taxa did appear to recover (Undibacterium and an ASV classified as Stenotrophomonas). These observations suggest that $\mathrm{Bd}$ infection may induce a shift to an alternate stable state, such that restoring the host to the uninfected state is insufficient to restore the microbiota to its initial state. Alternatively, recovery may occur but at a rate so slow as to be undetectable in the time frame of our experiment. Our results present an interesting contrast to a study of antiseptic-induced disturbance of the human skin microbiome, which documented marked disturbance followed by complete recovery within $6 \mathrm{~h}$ [61]. Both $\mathrm{Bd}$ infection and antiseptics represent strong disturbances, but resilience in these studies differed dramatically. The difference in resilience could reflect differences in the disturbance types or differences between human and amphibian skin such as permeability and the presence/absence of mucus glands and hair follicles.

We found that clearing $\mathrm{Bd}$ from the skin microbiome reduced among-frog variability in microbiome composition (i.e., reduces dispersion). In other words, clearance of $\mathrm{Bd}$ not only failed to reverse $\mathrm{Bd}$-induced changes in mean microbiome composition, but also reduced variability in composition. The effect on mean composition is illustrated by the degree of separation between the blue and red points in the ordination in Fig. $1 \mathrm{~B}$ and $\mathrm{C}$, while the effect on variability in composition is illustrated by the shrinking size of the confidence ellipse around $\mathrm{Bd}+$ individuals in Fig. 1C relative to Fig. 1B. This reduction in variability could be due to either the removal of $\mathrm{Bd}$ from the system, or the antifungal drug itself. Direct effects of the drug on the bacterial community are unlikely because itraconazole targets sterols, which are rarely produced by bacteria [62]. In addition, if the drug directly affected bacteria, we would expect to see the effect in all frogs, but we saw it only in $\mathrm{Bd}$ + frogs. However, drug treatment could indirectly affect the microbiome if it were to affect immune cell function, cause side effects in the frogs, or affect resident fungi besides $\mathrm{Bd}$ [46, 63]). Symbiotic fungi and bacteria can engage in facultative and inhibitory interactions, either directly or by influencing host immune responses, which could lead to changes in the bacterial community $[64,65]$. In this sense, drug treatment could be considered a second disturbance 
event to which these frogs are normally resistant (since Bdfrogs were not affected), but $\mathrm{Bd}$ erodes that resistance. Interestingly, it has been proposed that microbiome disturbance increases variability [66], but we observed the opposite. Increased dispersion might be expected if the disturbance compromises host ability to regulate the microbiome [66]. We hypothesize that, in the current study, reduced variability may instead have been a product of interactions within the microbial community due to the removal of Bd via mechanisms explained below.

Reduced microbial community dispersion might be due to $\mathrm{Bd}$ clearance per se, rather than drug treatment. We hypothesize that removing $\mathrm{Bd}$ creates vacancies in the microbial community, and that the set of bacteria most likely to occupy those vacancies is similar across frogs, leading to increased similarity among microbial communities. High similarity among frogs in the bacteria that colonize the open space could result from either selection or neutral processes. In the case of selection, certain bacteria might possess traits that allow them to take advantage of resources made available by the removal of $\mathrm{Bd}$, resulting in similar taxa filling the vacancies on most frogs. Alternatively, in a neutral framework, vacancies created by removal of Bd may be filled either through births within the local community (where the local community is the microbiome of one frog) or immigration from the metacommunity species pool, and community differences are not determined by species traits. In the absence of environmental gradients, the metacommunity species pool is assumed to be the same for all frogs. In this case, the sets of bacteria filling vacancies should vary randomly but not differ significantly among frogs, which could reduce among-frog microbiome variability. A previous study of $R$. sierrae indicated that $\mathrm{Bd}$ infection may affect immigration and/or drift in a taxon-specific manner [67]. Additional work is needed to determine the mechanism by which $\mathrm{Bd}$ clearance reduces microbial community variability. Notably, microbial composition and dispersion exhibited fundamentally different dynamics. Dispersion was unaffected by $\mathrm{Bd}$ infection, but strongly affected by Bd clearance. In contrast, composition changed due to $\mathrm{Bd}$ infection, and that effect largely persisted after Bd clearance. The ASVs that differed during the recovery period were generally a subset of those affected by Bd infection, suggesting that, in constrast to dispersion, composition did not experience a secondary shift due to Bd clearance.

It is possible that some degree of recovery occurs but was not detectable within the time span or sampling frequency of the experiment [12]. For example, it is possible that the degree of disturbance increased, followed by partial recovery, in the period after our post-infection sample, but this would not be detected without additional post-infection sampling points. However, our post-infection sample was collected on the date on which we measured the highest Bd infection loads $(P<$ 0.05 , Tukey adjusted), which we expect would limit increases in the magnitude of disturbance beyond our post-infection sampling point, minimizing unobserved recovery. It is also possible that the microbiome recovers very slowly and was therefore not detectable within the time frame of our experiment. While there are few data on amphibian microbiome resilience, one study showed that frog microbiomes disturbed by PIT tagging recovered in 2 weeks or fewer [14]. Another study of disturbance due to a related pathogen, while it did not formally analyze resilience, observed that three newts seemed to recover microbiome composition similar to controls, sometimes $<1$ week after clearing infection [15]. Furthermore, Loudon et al. [68] showed that transitioning salamanders to new environments resulted in marked shifts in microbiome composition within 1 week. These results suggest that the duration of our experiment would be sufficient to detect some level of resilience.

Captive and wild amphibians exhibit differences in skin microbiome composition [15, 17, 68], and resilience of wild frogs might differ from our observations. For example, it is possible that microbial species found only in natural environmental reservoirs promote resilience. However, studies show that environment does not overwhelm other drivers of microbiome structure: differences in the microbiota among amphibian species and populations persist even under shared environmental conditions [33,37], several core taxa are found in both lab and field settings [68], and microbial community responses to infection were consistent between lab and field settings [17], suggesting that laboratory studies can provide some insights into processes in nature.

Our study addresses resilience in microbiome structure composition but not function. Our finding that greater community compositional change corresponded to more negative change in body mass for the frogs is consistent with the hypothesis that resilience (return to the initial state) would be beneficial, but without demonstrating that the microbial change caused body mass loss, this conclusion is premature. Changes in microbiome structure do not always translate to change in community function, possibly due to functional redundancy among taxa. In addition, functional recovery may proceed at a different rate from taxonomic recovery [69]. Furthermore, disturbance to the microbial community does not necessarily translate to a detriment for the host: change to the microbiota following disease may erode function, but alternatively may indicate selective change toward a microbial community that is more resistant to Bd invasion (i.e., community adaptation to Bd). Studies that measure resistance to $\mathrm{Bd}$ during a second exposure (after the recovery period) would be needed to test whether microbiome changes are adaptive. Finally, the microbiome likely serves diverse functions beyond disease resistance, requiring untargeted assays such as functional shotgun 
metagenomic analyses, but these are rare for the amphibian skin microbiome (but see [70]). Future studies incorporating functional genetic data (e.g., metagenomics), or directly assessing functions of interest are critical for determining the functional significance of Bd-induced disturbances.

Our findings may provide insights for conservation strategies. There are no proven methods for controlling Bd outbreaks in wild populations. Managers and scientists are exploring the release of captive reared frogs, either through captive assurance or head starting programs, to support amphibian populations affected by Bd. These efforts can involve deliberate $\mathrm{Bd}$-exposure and clearance in attempts to immunize frogs prior to release [71]. Our results suggest that management interventions that expose amphibians to Bd could have lasting effects on the microbiota. Further research is needed to determine if this compositional shift is detrimental to microbiome function. If it is, managers may need to weigh the costs and benefits of immunization as part of conservation efforts.

\section{Data availability}

DNA sequence data have been deposited in the NCBI Sequence Read Archive under accession number PRJNA664873.

Acknowledgements The Sierra Nevada Aquatic Research Laboratory, San Francisco Zoo, National Park Service, and National Forest Service provided logistical support. Technical support and advanced computing resources from University of Hawaii Information Technology Services-Cyberinfrastructure are gratefully acknowledged. Carol S. Inatsuka provided graphical expertise. AJ Adams provided feedback on a previous version of this paper. This article is University of Hawai'i SOEST publication 11189. This research was supported by NSF award IOS-1455873 to AJJ and IOS-1457265 and DEB-1557190 to CJ Briggs and RAK.

\section{Compliance with ethical standards}

Conflict of interest The authors declare that they have no conflict of interest.

Publisher's note Springer Nature remains neutral with regard to jurisdictional claims in published maps and institutional affiliations.

Open Access This article is licensed under a Creative Commons Attribution 4.0 International License, which permits use, sharing, adaptation, distribution and reproduction in any medium or format, as long as you give appropriate credit to the original author(s) and the source, provide a link to the Creative Commons license, and indicate if changes were made. The images or other third party material in this article are included in the article's Creative Commons license, unless indicated otherwise in a credit line to the material. If material is not included in the article's Creative Commons license and your intended use is not permitted by statutory regulation or exceeds the permitted use, you will need to obtain permission directly from the copyright holder. To view a copy of this license, visit http://creativecommons. org/licenses/by/4.0/.

\section{References}

1. Connell JH. Diversity in Tropical Rain Forests and Coral Reefs. Science. 1978;199:1302-10.

2. Moreno-Mateos D, Barbier EB, Jones PC, Jones HP, Aronson J, López-López JA, et al. Anthropogenic ecosystem disturbance and the recovery debt. Nat Commun. 2017;8:14163.

3. Rodil IF, Lohrer AM, Chiaroni LD, Hewitt JE, Thrush SF. Disturbance of sandflats by thin terrigenous sediment deposits: consequences for primary production and nutrient cycling. Ecol Appl. 2011;21:416-26.

4. Carnell PE, Keough MJ. More severe disturbance regimes drive the shift of a kelp forest to a sea urchin barren in south-eastern Australia. Sci Rep. 2020;10:11272.

5. McDowell NG, Michaletz ST, Bennett KE, Solander KC, Xu C, Maxwell RM, et al. Predicting Chronic Climate-Driven Disturbances and Their Mitigation. Trends Ecol Evol. 2018;33:15-27.

6. Shade A, Peter H, Allison SD, Baho D, Berga M, Buergmann H, et al. Fundamentals of Microbial Community Resistance and Resilience. Front Microbiol. 2012;3:417.

7. Allison SD, Martiny JBH. Resistance, resilience, and redundancy in microbial communities. Proc Natl Acad Sci. 2008;105:11512-9.

8. Shade A, Read JS, Welkie DG, Kratz TK, Wu CH, McMahon KD. Resistance, resilience and recovery: aquatic bacterial dynamics after water column disturbance: Bacterial community recovery after lake mixing. Environ Microbiol. 2011;13:2752-67.

9. Shade A, Read JS, Youngblut ND, Fierer N, Knight R, Kratz TK, et al. Lake microbial communities are resilient after a wholeecosystem disturbance. ISME J. 2012;6:2153-67.

10. Dethlefsen L, Relman DA. Incomplete recovery and individualized responses of the human distal gut microbiota to repeated antibiotic perturbation. Proc Natl Acad Sci. 2011;108:4554-61.

11. Heinsen F-A, Knecht H, Neulinger SC, Schmitz RA, Knecht C, Kühbacher T, et al. Dynamic changes of the luminal and mucosaassociated gut microbiota during and after antibiotic therapy with paromomycin. Gut Microbes. 2015;6:243-54.

12. Fukuyama J, Rumker L, Sankaran K, Jeganathan P, Dethlefsen L, Relman DA, et al. Multidomain analyses of a longitudinal human microbiome intestinal cleanout perturbation experiment. PLOS Comput Biol. 2017;13:e1005706.

13. Subramanian S, Huq S, Yatsunenko T, Haque R, Mahfuz M, Alam MA, et al. Persistent gut microbiota immaturity in malnourished Bangladeshi children. Nature. 2014;510:417-21.

14. Antwis RE, Garcia G, Fidgett AL, Preziosi RF. Tagging Frogs with Passive Integrated Transponders Causes Disruption of the Cutaneous Bacterial Community and Proliferation of Opportunistic Fungi. Appl Environ Microbiol. 2014;80:4779-84.

15. Bates KA, Shelton JMG, Mercier VL, Hopkins KP, Harrison XA, Petrovan SO, et al. Captivity and Infection by the Fungal Pathogen Batrachochytrium salamandrivorans Perturb the Amphibian Skin Microbiome. Front Microbiol. 2019;10:1834.

16. Gimblet C, Meisel JS, Loesche MA, Cole SD, Horwinski J, Novais FO, et al. Cutaneous Leishmaniasis Induces a Transmissible Dysbiotic Skin Microbiota that Promotes Skin Inflammation. Cell Host Microbe. 2017;22:13-24.e4.

17. Jani AJ, Briggs CJ. The pathogen Batrachochytrium dendrobatidis disturbs the frog skin microbiome during a natural epidemic and experimental infection. Proc Natl Acad Sci. 2014;111:E5049-E5058.

18. Kong HH, Oh J, Deming C, Conlan S, Grice EA, Beatson MA, et al. Temporal shifts in the skin microbiome associated with disease flares and treatment in children with atopic dermatitis. Genome Res. 2012;22:850-9.

19. Longcore JE, Pessier AP, Nichols DK. Batrachochytrium Dendrobatidis gen. et sp. nov., a Chytrid Pathogenic to Amphibians. Mycologia. 1999;91:219-27. 
20. Berger L, Speare R, Daszak P, Green DE, Cunningham AA, Goggin CL, et al. Chytridiomycosis causes amphibian mortality associated with population declines in the rain forests of Australia and Central America. Proc Natl Acad Sci. 1998;95:9031-6.

21. Crawford AJ, Lips KR, Bermingham E. Epidemic disease decimates amphibian abundance, species diversity, and evolutionary history in the highlands of central Panama. Proc Natl Acad Sci. 2010;107:13777-82.

22. Lips KR, Brem F, Brenes R, Reeve JD, Alford RA, Voyles J, et al. Emerging infectious disease and the loss of biodiversity in a Neotropical amphibian community. Proc Natl Acad Sci USA. 2006;103:3165-70.

23. Vredenburg VT, Knapp RA, Tunstall TS, Briggs CJ. Dynamics of an emerging disease drive large-scale amphibian population extinctions. Proc Natl Acad Sci. 2010;107:9689-94.

24. Bletz MC, Loudon AH, Becker MH, Bell SC, Woodhams DC, Minbiole KPC, et al. Mitigating amphibian chytridiomycosis with bioaugmentation: characteristics of effective probiotics and strategies for their selection and use. Ecol Lett. 2013;16:807-20.

25. Hardy BM, Pope KL, Piovia-Scott J, Brown RN, Foley JE. Itraconazole treatment reduces Batrachochytrium dendrobatidis prevalence and increases overwinter field survival in juvenile Cascades frogs. Dis Aquat Organ. 2015;112:243-50.

26. McMahon TA, Sears BF, Venesky MD, Bessler SM, Brown JM, Deutsch K, et al. Amphibians acquire resistance to live and dead fungus overcoming fungal immunosuppression. Nature. 2014;511:224-7.

27. Harris RN, Brucker RM, Walke JB, Becker MH, Schwantes CR, Flaherty DC, et al. Skin microbes on frogs prevent morbidity and mortality caused by a lethal skin fungus. ISME J. 2009;3:818-24.

28. Muletz CR, Myers JM, Domangue RJ, Herrick JB, Harris RN. Soil bioaugmentation with amphibian cutaneous bacteria protects amphibian hosts from infection by Batrachochytrium dendrobatidis. Biol Conserv. 2012;152:119-26.

29. Becker MH, Harris RN, Minbiole KPC, Schwantes CR, RollinsSmith LA, Reinert LK, et al. Towards a Better Understanding of the Use of Probiotics for Preventing Chytridiomycosis in Panamanian Golden Frogs. Ecohealth. 2011;8:501-6.

30. Woodhams DC, Geiger CC, Reinert LK, Rollins-Smith LA, Lam B, Harris RN, et al. Treatment of amphibians infected with chytrid fungus: learning from failed trials with itraconazole, antimicrobial peptides, bacteria, and heat therapy. Dis Aquat Organ. 2012;98:11-25.

31. Belden LK, Hughey MC, Rebollar EA, Umile TP, Loftus SC, Burzynski EA, et al. Panamanian frog species host unique skin bacterial communities. Front Microbiol. 2015; 6:1171.

32. Bletz MC, Goedbloed DJ, Sanchez E, Reinhardt T, Tebbe CC, Bhuju S, et al. Amphibian gut microbiota shifts differentially in community structure but converges on habitat-specific predicted functions. Nat Commun. 2016;7:13699.

33. Jani AJ, Briggs CJ. Host and Aquatic Environment Shape the Amphibian Skin Microbiome but Effects on Downstream Resistance to the Pathogen Batrachochytrium dendrobatidis Are Variable. Front Microbiol. 2018;9:487.

34. Kueneman JG, Parfrey LW, Woodhams DC, Archer HM, Knight R, McKenzie VJ. The amphibian skin-associated microbiome across species, space and life history stages. Mol Ecol. 2014;23:1238-50.

35. Kueneman JG, Bletz MC, McKenzie VJ, Becker CG, Joseph MB, Abarca JG, et al. Community richness of amphibian skin bacteria correlates with bioclimate at the global scale. Nat Ecol Evol. 2019;3:381-9.

36. Küng D, Bigler L, Davis LR, Gratwicke B, Griffith E, Woodhams DC. Stability of Microbiota Facilitated by Host Immune Regulation: Informing Probiotic Strategies to Manage Amphibian Disease. PLoS ONE. 2014;9:e87101.
37. McKenzie VJ, Bowers RM, Fierer N, Knight R, Lauber CL. Cohabiting amphibian species harbor unique skin bacterial communities in wild populations. ISME J. 2012;6:588-96.

38. Prest TL, Kimball AK, Kueneman JG, McKenzie VJ. Hostassociated bacterial community succession during amphibian development. Mol Ecol. 2018;27:1992-2006.

39. Rebollar EA, Hughey MC, Medina D, Harris RN, Ibáñez R, Belden LK. Skin bacterial diversity of Panamanian frogs is associated with host susceptibility and presence of Batrachochytrium dendrobatidis. ISME J. 2016;10:1682-95.

40. Harrison XA, Price SJ, Hopkins K, Leung WTM, Sergeant C, Garner TWJ. Diversity-Stability Dynamics of the Amphibian Skin Microbiome and Susceptibility to a Lethal Viral Pathogen. Front Microbiol. 2019;10:2883.

41. Jani AJ, Knapp RA, Briggs CJ. Epidemic and endemic pathogen dynamics correspond to distinct host population microbiomes at a landscape scale. Proc R Soc B-Biol Sci. 2017;284:20170944.

42. Walke JB, Becker MH, Loftus SC, House LL, Teotonio TL, Minbiole KPC, et al. Community Structure and Function of Amphibian Skin Microbes: an Experiment with Bullfrogs Exposed to a Chytrid Fungus. PLOS ONE. 2015;10:e0139848.

43. Knutie SA, Wilkinson CL, Kohl KD, Rohr JR. Early-life disruption of amphibian microbiota decreases later-life resistance to parasites. Nat Commun. 2017;8:86.

44. Rachowicz LJ, Knapp RA, Morgan JA, Stice MJ, Vredenburg VT, Parker JM, et al. Emerging infectious disease as a proximate cause of amphibian mass mortality. Ecology. 2006;87:1671-83.

45. Jones MEB, Paddock D, Bender L, Allen JL, Schrenzel MD, Pessier AP. Treatment of chytridiomycosis with reduced-dose itraconazole. Dis Aquat Organ. 2012;99:243-9.

46. Brannelly LA. Reduced Itraconazole Concentration and Durations Are Successful in Treating Batrachochytrium dendrobatidis Infection in Amphibians. JOVE-J Vis Exp. 2014;85:e51166.

47. Hyatt AD, Boyle DG, Olsen V, Boyle DB, Berger L, Obendorf D, et al. Diagnostic assays and sampling protocols for the detection of Batrachochytrium dendrobatidis. Dis Aquat Organ. 2007;73:175-92.

48. Boyle DG, Boyle DB, Olsen V, Morgan JAT, Hyatt AD. Rapid quantitative detection of chytridiomycosis (Batrachochytrium dendrobatidis) in amphibian samples using real-time Taqman PCR assay. Dis Aquat Organ. 2004;60:141-8.

49. Kozich JJ, Westcott SL, Baxter NT, Highlander SK, Schloss PD. Development of a Dual-Index Sequencing Strategy and Curation Pipeline for Analyzing Amplicon Sequence Data on the MiSeq Illumina Sequencing Platform. Appl Environ Microbiol. 2013;79: 5112-20.

50. Klindworth A, Pruesse E, Schweer T, Peplies J, Quast C, Horn M, et al. Evaluation of general $16 \mathrm{~S}$ ribosomal RNA gene PCR primers for classical and next-generation sequencing-based diversity studies. Nucleic Acids Res. 2013;41:e1-e1.

51. Callahan BJ, McMurdie PJ, Rosen MJ, Han AW, Johnson AJA, Holmes SP. DADA2: High-resolution sample inference from Illumina amplicon data. Nat Methods. 2016;13:581-3.

52. Schloss PD, Westcott SL, Ryabin T, Hall JR, Hartmann M, Hollister EB, et al. Introducing mothur: Open-Source, PlatformIndependent, Community-Supported Software for Describing and Comparing Microbial Communities. Appl Environ Microbiol. 2009;75:7537-41.

53. Quast C, Pruesse E, Yilmaz P, Gerken J, Schweer T, Yarza P, et al. The SILVA ribosomal RNA gene database project: improved data processing and web-based tools. Nucleic Acids Res. 2013;41: D590-596.

54. Frøslev TG, Kjøller R, Bruun HH, Ejrnæs R, Brunbjerg AK, Pietroni C, et al. Algorithm for post-clustering curation of DNA amplicon data yields reliable biodiversity estimates. Nat Commun. 2017;8:1188. 
55. Arisdakessian C, Cleveland SB, Belcaid M. MetaFlowlmics: Scalable and Reproducible Nextflow Pipelines for the Analysis of Microbiome Marker Data. Pract Exp Adv Res Comput. 2020. Association for Computing Machinery, New York, NY, USA, pp 120-4.

56. Lozupone C, Knight R. UniFrac: a New Phylogenetic Method for Comparing Microbial Communities. Appl Environ Microbiol. 2005;71:8228-35.

57. Anderson MJ. A new method for non-parametric multivariate analysis of variance. Austral Ecol. 2001;26:32-46.

58. Anderson MJ. Permutational Multivariate Analysis of Variance (PERMANOVA). Wiley statsref: statistics reference online. American Cancer Society;2017. p. 1-15.

59. Segata N, Izard J, Waldron L, Gevers D, Miropolsky L, Garrett WS, et al. Metagenomic biomarker discovery and explanation. Genome Biol. 2011;12:R60.

60. Joseph MB, Knapp RA. Disease and climate effects on individuals jointly drive post-reintroduction population dynamics of an endangered amphibian. bioRxiv. 2018; 332114.

61. SanMiguel AJ, Meisel JS, Horwinski J, Zheng Q, Bradley CW, Grice EA. Antiseptic Agents Elicit Short-Term, Personalized, and Body Site-Specific Shifts in Resident Skin Bacterial Communities. J Investig Dermatol. 2018;138:2234-43.

62. Volkman J. Sterols in microorganisms. Appl Microbiol Biotechnol. 2003;60:495-506.

63. Niño DF, Cauvi DM, De Maio A. Itraconazole, a Commonly Used Antifungal, Inhibits Fc $\gamma$ Receptor-Mediated Phagocytosis: Alteration of Fcy Receptor Glycosylation and Gene Expression. Shock. 2014;42:52.

64. Tang C, Kamiya T, Liu Y, Kadoki M, Kakuta S, Oshima K, et al. Inhibition of Dectin-1 Signaling Ameliorates Colitis by Inducing
Lactobacillus-Mediated Regulatory $\mathrm{T}$ Cell Expansion in the Intestine. Cell Host Microbe. 2015;18:183-97.

65. Zuo T, Wong SH, Cheung CP, Lam K, Lui R, Cheung K, et al. Gut fungal dysbiosis correlates with reduced efficacy of fecal microbiota transplantation in Clostridium difficile infection. Nat Commun. 2018;9:3663.

66. Zaneveld JR, McMinds R, Vega Thurber R. Stress and stability: applying the Anna Karenina principle to animal microbiomes. Nat Microbiol. 2017;2:17121.

67. Wilber MQ, Jani AJ, Mihaljevic JR, Briggs CJ. Fungal infection alters the selection, dispersal and drift processes structuring the amphibian skin microbiome. Ecol Lett. 2019;23:88-98.

68. Loudon AH, Woodhams DC, Parfrey LW, Archer H, Knight R, McKenzie V, et al. Microbial community dynamics and effect of environmental microbial reservoirs on red-backed salamanders (Plethodon cinereus). ISME J. 2013;8:830-40.

69. Santillan E, Constancias F, Wuertz S. Press Disturbance Alters Community Structure and Assembly Mechanisms of Bacterial Taxa and Functional Genes in Mesocosm-Scale Bioreactors. mSystems. 2020;5:e00471-20.

70. Rebollar EA, Gutiérrez-Preciado A, Noecker C, Eng A, Hughey MC, Medina D, et al. The Skin Microbiome of the Neotropical Frog Craugastor fitzingeri: inferring Potential Bacterial-HostPathogen Interactions From Metagenomic Data. Front Microbiol. 2018;9:466.

71. Mountain Yellow-legged Frog Interagency Technical Team. Interagency Conservation Strategy for Mountain Yellow-legged Frogs in the Sierra Nevada (Rana sierrae and Rana muscosa). Version 1. California Department of Fish and Wildlife, National Park Service, U.S. Fish and Wildlife Service, U.S. Forest Service; 2018. 